\title{
99. Effect of Diameter on Notch-Coefficient of Rotary Bending Test Piece.
}

\author{
By Akimasa O No, M.J.A. \\ (Comm. Oct. 12, 1951.)
}

\section{Introductory.}

This note aims at analysing the results of rotary bending tests made several years ago by Mr. A. Tagawa, then a Member of the Research Laboratories of Yawata Steel works. The object of the test was to find the effect of specimen-size on endurance limits of carbon steel and on notch-coefficient in particular. The test was made chiefly in a rotary bending machine specially made for testing specimens of diameter up to some $70 \mathrm{~mm}$ and partly in usual smaller machine, both machines being of uniform moment type. When used for small specimens, the former gave results concordant with the latter - a result confirmed previously. Though the plan of the test was rather limited, the analysis gives a clue to formulating the coefficient in term of the theoretical factor.

\section{Endurance Tests.}

Materials tested were ordinary carbon steels with the chemical composition and mechanical properties as shown in Table $1^{*}$.

Table 1. Chemical Analysis and Tensile Test.

\begin{tabular}{|c|cccccc|cccc|}
\hline Series & C & Si & Mn & P & S & Cu & $\begin{array}{c}\text { Tensile } \\
\text { Strength } \\
\mathrm{kg} / \mathrm{mm}^{2}\end{array}$ & $\begin{array}{c}\text { Yield } \\
\text { Point } \\
\mathrm{kg} / \mathrm{mm}^{2}\end{array}$ & $\begin{array}{c}\text { Elongation cent } \\
\text { per cont }\end{array}$ & $\begin{array}{c}\text { Area } \\
\text { per cent }\end{array}$ \\
& 0.26 & 0.10 & 0.43 & 0.06 & 0.05 & 0.14 & 44 & 21 & 34 & 49 \\
$\mathrm{G}$ & 0.36 & 0.23 & 0.71 & 0.05 & 0.04 & 0.15 & 56 & 32 & 29 & 54 \\
$\mathrm{H}$ & 0.38 & 0.14 & 0.47 & 0.024 & 0.056 & 0.036 & 53 & 31 & 28 & 52 \\
\hline
\end{tabular}

Test pieces for endurance tests were taken from rolled bars, $80 \mathrm{~mm}$ (Ser. $\mathrm{F}$ and $\mathrm{G}$ ) and $90 \mathrm{~mm}$ (Ser. $\mathrm{H}$ ) in diameters; they were prepared by cutting after normalizing. Particularly, small test pieces of diameters $d=10$ and $12 \mathrm{~mm}$ were taken from different depths of the original bars; the position is denoted in Table 2 by the diameter of the circle drawn through the centre of the testpiece-section. Larger pieces were all concentric with the original bars. The diameter $d$ of notched specimens is that of a minimum section through the bottom of the notch-groove surrounding the piece.

The profile of the notch-section, Fig. 1, consists of two straight lines making an angle of $55 \mathrm{deg}$. with each other and connected by

* Tables 1-3 are due to Tagawa. 


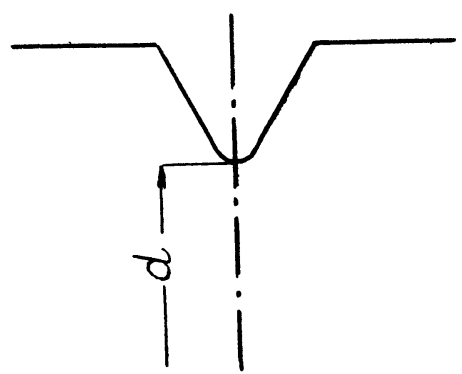

Fig. 1.

an arc of radius $0.029 \mathrm{~d}$; the depth of the notch is $0.135 \mathrm{~d}$. The theoretical factor of stress-concentration or the ratio of the peak stress to the nominal stress (bending moment divided by section modulus) is denoted by $\alpha$, of which the numerical value found by using Neuber's formulae, namely by combining the factors for a deep hyperbolic notch and a notch with a finite depth, is 3 with the Poisson's ratio 0.3 .

Table 2. Endurance Limits of Stresses $\sigma$ and p.

\begin{tabular}{|c|c|c|c|c|}
\hline Series & $\begin{array}{c}\text { Diameter } \\
\text { of Test } \\
\text { Piece } \\
\text { d } \\
\mathrm{mm}\end{array}$ & $\begin{array}{c}\text { Diameter of } \\
\text { Circle thr. } \\
\text { Centre of } \\
\text { Sp. Sect. } \\
\text { mm }\end{array}$ & $\begin{array}{c}\text { Bending Stress } \\
\text { in Unnotched } \\
\text { Specimen } \\
\sigma \\
\mathrm{kg} / \mathrm{mm}\end{array}$ & $\begin{array}{c}\text { Nominal Stress } \\
\text { in Notched } \\
\text { Specimen } \\
\text { p } \\
\mathrm{kg} / \mathrm{mm}^{2}\end{array}$ \\
\hline \multirow[t]{4}{*}{$\mathbf{F}$} & 12 & 50 & 23 & - \\
\hline & 12 & 50 & 23 & 9.5 \\
\hline & 24 & 0 & 21.5 & 9 \\
\hline & 50 & 0 & 20 & 8 \\
\hline \multirow[t]{8}{*}{$\mathbf{G}$} & 12 & 60 & 27 & 12 \\
\hline & 12 & 48 & 27 & - \\
\hline & 12 & 36 & 26 & 12 \\
\hline & $12^{\dagger}$ & 24 & 26 & - \\
\hline & $12^{\dagger}$ & 12 & 25 & - \\
\hline & 36 & 0 & 24.5 & 9.5 \\
\hline & 48 & 0 & 24 & 9.5 \\
\hline & 60 & 0 & 23 & $9 *$ \\
\hline \multirow[t]{5}{*}{$\mathbf{H}$} & 10 & 70 & 25 & - \\
\hline & 10 & 60 & 24 & - \\
\hline & 10 & 48 & 25 & - \\
\hline & 48 & 0 & 23 & - \\
\hline & 70 & 0 & 20 & - \\
\hline
\end{tabular}

Remarks: $†$ Specimens are contained in an area corresponding to the cross section of a greater specimen.

* This figure depends partly on the judgement, the $\mathrm{p}-\mathrm{N}$ (stress vs. number of cycles) relation being irregular.

Table 2 shows the stresses endured by notched and unnotched specimens to the number of stress-cycles $\mathrm{N}=10^{7}$; in the table $\sigma$ is the bending stress at the surface of unnotched specimens and $p$ is the nominal stress at the bottom of notch. The material for Ser. $\mathrm{G}$ is not quite uniform as is revealed by $\sigma$ for $\mathrm{d}=12 \mathrm{~mm}$. 


\section{Discussion of the Results.}

In unnotched specimens it is seen that $\sigma$ falls with the rise of

d. When expressed as

$$
\sigma=\sigma^{*}\left(1+\frac{\varepsilon}{d}\right)
$$

the values of $\sigma^{*}$ and $\varepsilon$ are as follows:

\begin{tabular}{crrlrl} 
Series & \multicolumn{1}{c}{$F$} & \multicolumn{2}{c}{$\mathrm{G}$} & $\mathrm{H}$ & \\
$\sigma^{*}$ & 19.3 & 22.9 & $(22.8)$ & 20.8 & $\mathrm{~kg} / \mathrm{mm}^{2}$, \\
$\varepsilon$ & 2.3 & 1.7 & $(2.1)$ & 1.9 & $\mathrm{~mm}$,
\end{tabular}

$\mathrm{d}$ being measured in $\mathrm{mm}$. The values in the brackets were found by excluding two specimens marked with $\uparrow$ in Table 2 . The standard error of $\varepsilon$ for $F$ and $G$ is $\pm 0.3- \pm 0.4$ (the latter refers to $\varepsilon=1.7$ for $G$ ), but that for $H$ amounts to $\pm 0.65, \varepsilon$ being less significant as compared with those for other series.

Now the notch-coefficient is found by $\beta=\frac{\sigma}{p}$. The value of $\beta$ is definite when the diameters of specimens are specified. In Table 3 two series of $\beta$ are given; in one series notched and unnotched specimens of equal diameters are compared and in the other $12 \mathrm{~mm}$ diameter is taken throughout for unnotched specimens. To classify these two series, $\beta^{\prime}$ is written momentarily for $\beta$ of the second series.

Table 3. Values of $\alpha, \beta$, and $\beta^{\prime}$.

\begin{tabular}{|c|c|c|c|c|c|}
\hline Series & $\begin{array}{l}\text { Diameter of } \\
\text { Test Piece } \\
\text { d mm }\end{array}$ & & $\begin{array}{l}\text { Factor } \\
\quad \alpha\end{array}$ & $\begin{array}{c}\text { Notch-Coefficient, } \\
\text { Equal Diams. } \\
\beta\end{array}$ & $\begin{array}{l}\text { Notch-Coefficient, } \\
12 \mathrm{~mm} \text { Base } \\
\boldsymbol{\beta}^{\prime}\end{array}$ \\
\hline \multirow[t]{4}{*}{$F$} & 12 & & 3.668 & 2.42 & 2.42 \\
\hline & 24 & & 2.974 & 2.39 & 2.56 \\
\hline & 50 & & 3.042 & 2.50 & 2.88 \\
\hline & \multicolumn{2}{|c|}{ Mean } & 3.028 & 2.44 & - \\
\hline \multirow[t]{7}{*}{ G } & 12 & & 3.051 & 2.25 & 2.25 \\
\hline & 12 & & 3.040 & 2.17 & 2.17 \\
\hline & 36 & & 3.108 & 2.58 & $2.74(26 / 9.5)$ \\
\hline & 48 & & 3.039 & 2.53 & $2.84(27 / 9.5)$ \\
\hline & 60 & & 2.976 & 2.56 & $3.00(2 \pi / 9)$ \\
\hline & \multicolumn{2}{|c|}{ Mean } & 3.043 & 2.42 & - \\
\hline & \multicolumn{2}{|c|}{ Total Mean } & 3.037 & 2.43 & - \\
\hline
\end{tabular}

First it is found that the difference between the two mean values of $\alpha$ is insignificant. Next as for $\beta$, the mean values of the respective series are nearly equal. Really, examining the difference between the two values in comparison with the standard error, it is found with reference to the $t$-values that the difference is hardly 
significant. So the two series may be taken together for testing the regression of $\beta$ on $\frac{1}{d}$, namely by the formula:

$$
\beta=a+b(x-\bar{x}), \quad x=\frac{1}{d} .
$$

The values of the constants are $a=2.425$ and $b=-4.11 \mathrm{~mm}$. The latter constant is found to be quite significant when compared with zero. For the sake of convenience $\beta$ is written in the form :

$$
\beta=\beta^{*}\left(1-\frac{\delta}{d}\right)
$$

with $\beta^{*}=2.62 \pm 0.06$ and $\delta=1.6 \pm 0.4 \mathrm{~mm}$ ( $d$ also in $\mathrm{mm}$ ).

Lastly, $\beta^{\prime}$ given in the last column of Table 3 is seen to increase with the diameter; this point will be specially considered in the following.

\section{Interpretation of $\beta^{\prime}$.}

To interpret $\beta^{\prime}$ in terms of $\sigma$ and $\beta$, a notation is used for signifying the diameter of specimens. For example, let $\sigma(d)$ represent $\sigma$ for a diameter $d$. Then, taking a standard diameter $d_{0}$ for unnotched specimen $-12 \mathrm{~mm}$ in the present case, the coefficient $\beta^{\prime}$ for the notched specimen of a diameter $d$ is

$$
\beta^{\prime}=\frac{\sigma\left(d_{n}\right)}{p(d)}=\beta(d) \frac{\sigma\left(d_{n}\right)}{\sigma(d)},
$$

since $p(d)=\frac{\sigma(d)}{\beta(d)}$; that is, by substituting (1) and (2) for $\sigma$ and $\beta$,

$$
\beta^{\prime}=\beta^{*}\left(1+\frac{\varepsilon}{d_{0}}\right)\left(1-\frac{\delta}{d}\right) /\left(1+\frac{\varepsilon}{d}\right) \text {. }
$$

So far the interpretation is made on the experimental basis. Apart from any experimental error, (3) is acceptable in logical sense. The next thing, however, is to express $\beta^{*}$ in $\alpha$. For this purpose use is made of a theory of strength (s. Appendix), which is thought to be in accord with experience when the stress-distribution is more or less even. As $\beta^{*}$ refers to a great diameter and therefore to a small stress-gradient, the theory applies and it will be shown in (8) that $\beta^{*}=\mu \alpha$, where $\mu$ is a coefficient representing the effect of the second principal stress acting in the circumferential direction of the notch-groove. Giving to $\mu$ an estimated value 0.9 , $\mu \alpha=2.73$ for $\alpha=3.037$. This being compared with $\beta^{*}=2.62$, the difference is scarcely significant; the above value of $\mu$ is taken partly in view of this point, to say nothing of the attention paid to the material quality. In (3) we may put $\varepsilon=2.0 \mathrm{~mm}$ (a mean value of $F$ and $G$ ) and $\delta=1.6 \mathrm{~mm}$. With $d_{0}=12 \mathrm{~mm}$ and $\mu=0.9$ as stated above, the expression is equal to $1.05 \alpha$ multiplied by a factor 
which is less than unity for a finite value of $d$ and increases with $d$ tending to unity in the limit.

\section{Concluding Remark.}

The regression of $p$ on $\frac{1}{d}$ was not directly found in the above because of the difference between Ser. $F$ and $G$ existing in regard to the values of $p$. If it could be found, however, the result would be of the form:

$$
p(d)=p^{*}\left(1+\frac{c}{d}\right), \quad c>0
$$

as this expression is about the same in a definite range of $d$ as (1) and (2) combined. Then, remembering that $\sigma^{*}=\beta^{*} p^{*}$, it is seen that

$$
\beta^{\prime}=\beta^{*}\left(1+\frac{\varepsilon}{d_{0}}\right) /\left(1+\frac{c}{d}\right)
$$

or with $k=\mu\left(1+\frac{\varepsilon}{d_{0}}\right)$

$$
\beta^{\prime}=\frac{\alpha k}{1+\frac{c}{d}} .
$$

Referring to (1) and (2), we may take $c=$ some $3.9 \mathrm{~mm}$ for $d=12-$ $60 \mathrm{~mm}$. To apply (4) to other cases in general, it is to be noted that $\varepsilon$ depends on the quality of material, while $c$ depends not only on the material quality but probably also on the value of $\alpha$.

\section{Appendix-Note on Theory of Strength.}

In Mohr's stress diagram, Fig. 2, the critical line of rupture is represented by a straight line defined by the length and incli-

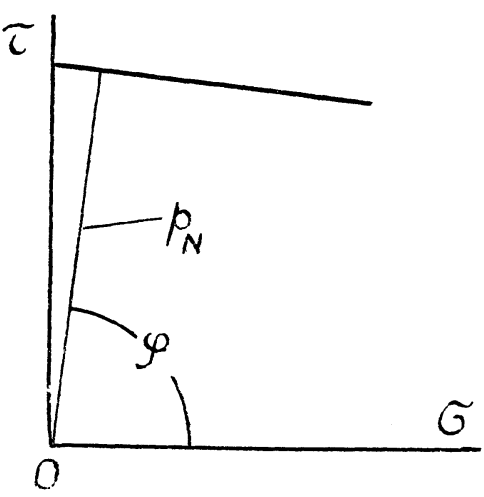

Fig. 2. nation of the perpendicular drawn from the origin. The length $p_{N}$ is considered to vary with the action of stress-static or vibratory-before rupture taking place. In the present case static stress is absent; so we write simply

$$
p_{N}=p_{0}-\alpha_{N} R,
$$

where $p_{0}$ is a constant, $\alpha_{N}$ a parameter depending on number of cycles $N$, and

$$
R=\left[\left(\sigma_{1}-\sigma_{2}\right)^{2}+\left(\sigma_{2}-\sigma_{3}\right)^{2}+\left(\sigma_{1}-\sigma_{3}\right)^{2}\right]^{\frac{1}{2}},
$$


$\sigma_{1}, \sigma_{2}, \sigma_{3}$ being vibratory principal stresses, $\sigma_{1}>\sigma_{2}>\sigma_{3}$. Then, the condition of rupture is given by

$$
\frac{1}{2}\left(\sigma_{1}-\sigma_{3}\right)+\frac{1}{2}\left(\sigma_{1}+\sigma_{3}\right) \cos \varphi=p_{0}-\alpha_{N} R,
$$

provided that the stress-gradient is small-if not, stress at a point is not sufficient to define the strength.

So restricting our view to large specimens, we have to compare the critical conditions in the two cases, namely: unnotched specimens, $\sigma_{1}=\sigma^{*}, \sigma_{2}=\sigma_{3}=0$; notched specimens, $\sigma_{1}=\alpha p^{*}, \sigma_{2}=n \sigma_{1},(n<1)$, and $\sigma_{3}=0$. In these cases (6) says that

$$
\frac{1}{2} \sigma^{*}\left[1+\cos \varphi+\alpha_{v} 2 \sqrt{2}\right]=p_{0}
$$

and

$$
\frac{1}{2} \alpha p^{*}\left[1+\cos q+\alpha_{N} 2 \sqrt{2} \sqrt{1-n+n^{2}}\right]=p_{0},
$$

respectively. Eliminating $p_{0}$ from these two equations and putting

$$
c^{\prime}=\frac{1+\cos \phi}{1+\cos \varphi+\alpha_{N} 2 \sqrt{2}}
$$

it is seen that, since $\frac{\sigma^{*}}{p^{*}}=\beta^{*}$,

where

$$
\beta^{*}=\mu \alpha
$$

$$
\mu=c^{\prime}+\left(1-c^{\prime}\right) \sqrt{1-n+n^{2}} .
$$

When the Poisson's ratio is 0.3 , the value of $n$ for a deep notch is found to be 0.31 ; using this value invariably for the present calculation, we find that $\sqrt{1-n+n^{2}}=0.887$. Next, the numerical values of $\alpha_{N}$ and $\cos \phi$ found from some data of combined stress experiments published in the literature show that in ductile materials $\cos \phi$ is small and $\alpha_{N}$ large in contrast with brittle materials. This condition speaks, according to (7), for a small value to be taken for $c^{\prime}$ in the present case. This being so, the value of $\mu$ varies a little, namely, from 0.887 to 1 as $c^{\prime}$ varies from 0 to 1 . It is thought that when we take tentatively $\mu=0.9$ for short by disregarding the second figure of decimals, the error is probably within a few per cent. 